\title{
Meal habits and metabolic status in Southern Italian adults
}

\author{
Gaetano Zappala ${ }^{\mathrm{a}}$, Armando Platania ${ }^{\mathrm{a}}$, Grazia Paladino ${ }^{\mathrm{a}}$, Liberata Keti Nicolosi ${ }^{\mathrm{a}}$, \\ Rosalia Ragusa $^{\mathrm{b}}$ and Marina Marranzano ${ }^{\mathrm{a}, *}$ \\ a Department of Medical and Surgical Sciences and Advanced Technologies “G.F. Ingrassia”, \\ University of Catania, Catania, Italy \\ bClinical Directorate, University Hospital “G. Rodolico” Catania, Catania, Italy
}

\begin{abstract}
.
BACKGROUND: Recently eating habits, such as breakfast, snaking, and out-of-home meals, have been shown to influence metabolic health of general population.

OBJECTIVE: The aim of this study is to explore the association between meal habits and metabolic status in Southern Italian adults.

METHODS: Data from the Mediterranean healthy Eating, Aging and Lifestyle study was used, including a total of 1952 subjects.

RESULTS: Individuals having type 2 diabetes, dyslipidemias, and hypertension were more likely to adhere to a diet characterized by skipping breakfast, having unhealthy snacks and having out-of-home meals. Having breakfast was associated with higher odds of having dyslipidemias ( $\mathrm{OR}=2.71,95 \% \mathrm{CI}: 1.74,4.23)$; having snacks was associated with higher odds of being obese ( $\mathrm{OR}=2.09,95 \% \mathrm{CI}: 1.42,3.09)$; and having out-of-home meals was associated with higher odds of having dyslipidemias ( $\mathrm{OR}=1.55,95 \% \mathrm{CI}: 1.03,2.32)$ and hypertension $(\mathrm{OR}=1.61,95 \%$ CI: $1.18,2.21)$.
\end{abstract}

CONCLUSIONS: The results of this study indicate that eating habits may be associated with metabolic status in adults living in the Mediterranean area.

Keywords: Snack, metabolic health, Mediterranean, breakfast, hypertension, diabetes, obesity

\section{Introduction}

Several studies show a worldwide progressive increase in body mass index (BMI) and in the prevalence of overweight and obesity [1]. Obese people have higher risk of other metabolic disorders, including hypertension, diabetes, dyslipidemia, which in turn increase the risk for non-communicable diseases (NCDs) including cardiovascular diseases (CVDs) and some cancers [2]. Dietary habits have been considered crucial in preventing such conditions: besides diet quality and healthy dietary patterns [3], and individual foods groups and beverages such as fruit and vegetable [4], nuts [5], dairy products [6], egg [7], and

\footnotetext{
*Corresponding author: Marina Marranzano, Department of Medical and Surgical Sciences and Advanced Technologies "G.F. Ingrassia”, University of Catania, Catania, Italy. Tel.: 0953782180; E-mail:marranz@unict.it.
}

coffee [8], also eating habits have been studied in relation to metabolic disorders. In fact, change in eating habits, such as consumption of breakfast, increase in snacking and meals outside the home have been associated, with a various extent, to modification in weight status in adults and younger generations $[9,10]$. The increase in trade and consumption of junk food may have contributed to the increase in the prevalence of obesity, especially among adolescent $[11,12]$. Several studies have shown that a greater consumption of snacks could lead to a reduction in the consumption of regular meals or even higher tendency to skip meals with important consequences for health due to the lower food diversity and lower intake of nutrients, as healthier food choices and a better intake of macronutrients and micronutrients are associated with regular meals $[11,12]$. Other studies carried out in recent years and conducted in most cases in the USA, show 
that there is a correlation between the frequency and type of out of home eating with the increase in BMI and the accumulation of visceral fat [13-16]. Considering the available evidence regarding unhealthy dietary choices and health, we aimed to investigate the association between meal habits and metabolic status in Southern Italian adults.

\section{Methods}

\subsection{Study design and population}

The MEAL (Mediterranean Healthy Eating, Ageing, and Lifestyle) study is an observational study aiming to investigate nutritional habits and their relation with lifestyle behaviors characterizing the classical Mediterranean lifestyle. The baseline data comprised a total sample of 2044 men and women $>18$ years old, stratified by gender and 10year age groups, randomly selected in the main districts of the city of Catania, southern Italy. The enrolment and data collection was performed between the years 2014 and 2015, with the extraction of random subjects from the lists of a pool of general practitioners' registered patients. Detailed information on the study protocol is published elsewhere [17]. All participants were informed about the aims of the study and provided a written informed consent. All the study procedures were carried out in accordance with the Declaration of Helsinki (1989) of the World Medical Association. The study protocol has been approved by the concerning ethical committee.

\subsection{Data collection}

An electronic data collection was performed by face-to-face computer-assisted personal interview using tablet computers. Participants were provided with a paper copy of the questionnaire to visualize the response options, but the final answers were filled by the interviewer directly on the digital device (tablet computer). The demographic data included gender, age at recruitment, highest educational degree achieved, occupation (specifies the nature of the most important employment during the year before the investigation) or last occupation before retirement, and marital status. Educational status was divided in the following categories: (i) low (primary/secondary), (ii) medium (high school), and (iii) high (university). Occupational status was categorized as (i) unemployed, (ii) low (unskilled workers), (iii) medium (partially skilled workers) and (iv) high (skilled workers). Physical activity status was evaluated using the International Physical Activity Questionnaires (IPAQ) [27], which comprised a set of questionnaires (five domains) investigating the time spent being physically active in the last 7 days: based on the IPAQ guidelines, the final scores allows to categorized physical activity level as (1) low, (2) moderate, and (3) high. Smoking status was categorized as (1) non-smoker, (2) ex-smoker, and (3) current smoker. Alcohol consumption was categorized as (1) none, (2) moderate drinker (0.1-12 g/day), and (3) regular drinker (>12 g/day).

\subsection{Dietary assessment}

Food intake was evaluated using a validated food frequency questionnaire FFQ that have been already tested for validity and reliability for the Sicilian population $[18,19]$. Following the identification of the food intake, the energy content as well as the macroand micro-nutrient intakes [20,21] were obtained through comparison with food composition tables of the Research Center for Foods and Nutrition. Intake of seasonal foods refers to consumption during the period in which the food was available and then adjusted by its proportional intake in one year. FFQs with unreliable intakes $(<1000$ or $>6000 \mathrm{kcal} / \mathrm{d})$ were excluded from the analyses $(n=92)$ leaving a total of 1952 individuals included in the analysis.

\subsection{Eating habits}

Participants were asked a set of questions describing their eating habits. Specifically, participants were asked how many times per week, on average, they usually have breakfast and, regarding out-of-home meals, they usually have a main meal (lunch or dinner) not eaten at home. Moreover, participants were also asked how many times per day, on average, they usually have snacks.

\subsection{Anthropometric measurements and metabolic variables}

Anthropometric examinations were collected using standardized methods. Height was measured to the nearest $0.5 \mathrm{~cm}$ without shoes, with the back square against the wall tape, eyes looking straight ahead, with a right-angle triangle resting on the scalp and 
against the wall. Body mass index (BMI) was calculated, and patients were categorized as under/normal weight (BMI $<25 \mathrm{Kg} / \mathrm{m} 2$ ), overweight (BMI 25 to $29.9 \mathrm{Kg} / \mathrm{m} 2$ ), and obese (BMI $>30 \mathrm{Kg} / \mathrm{m} 2)$. Waist circumference (centimeters) was measured midway between the 12th rib and the iliac crest and hip circumference (centimeters) around the buttocks, at the level of the maximum extension. Waist-to-hip ratio was then calculated. Arterial blood pressure was measured at the end of the physical examination with subject in sitting position and at least 5 minutes at rest. Blood pressure measurements was taken three times at the right arm relaxed and well supported by a table, with an angle of $45^{\circ}$ from the trunk. A mean of the last two measurements was considered for inclusion in the database. Information from measurements was integrated with general practitioners computerized records, as a specialist diagnoses patients with disease in order to obtain drug. Patients have been considered hypertensive when average systolic/diastolic blood pressure levels were greater or equal to $140 / 90 \mathrm{~mm}$ $\mathrm{Hg}$, taking anti-hypertensive medications, or being previously diagnosed of hypertension; patients were considered diabetic or having dyslipidemia whether previously diagnosed of diabetes and hypercholesterolemia/hypertriglyceridemia, respectively.

\subsection{Statistical analysis}

Frequencies are presented as absolute numbers and percentages; continuous variables are presented as means and standard deviations. Differences between groups were compared with Chi-square test for categorical variables, Student's $t$-test and ANOVA for continuous variables distributed normally, and Mann-Whitney U-test and Kruskall-Wallis test for variables distributed not normally. Multivariate logistic regression analyses were used to test association between eating habits and metabolic outcomes, adjusting for potential confounding factors. All reported $P$ values were based on two-sided tests and compared to a significance level of 5\%. SPSS 17 (SPSS Inc., Chicago, IL, USA) software was used for all the statistical calculations.

\section{Results}

Baseline characteristics by breakfast, snacking, and out-of-home eating are presented in Table 1. Significant differences occurred for age groups (a lower proportion of younger individuals consumed regularly breakfast and snacks, while a higher proportion had regular out-of-home meals), sex (a higher proportion of women reported having breakfast and snacks while a lower proportion reporting having outof-home meals), educational and occupational status (a higher proportion of lower categories reported consuming breakfast, while a higher proportion of higher categories reported having snacks and out-ofhome meals), and smoking status (a higher proportion of non-smokers consumed regularly breakfast and snacks, while a higher proportion of ex-smokers had regular out-of-home meals). Differences in marital status regarded only out-of-home eating (a higher proportion of singles reported consuming out-ofhome meals). Snacks and out-of-home meals were consumed by a higher proportion of individuals having moderate to high physical activity levels, while a higher proportion of moderate alcohol consumption was reported among breakfast skippers and out-ofhome eaters.

Table 1 also report the differences in frequency of metabolic disorders by meal habits: no difference in distribution of overweight individuals was found, but a higher and a lower prevalence of obese was observed among those eating snacks and having out-of-home meals, respectively. Among those having type 2 diabetes, dyslipidemias, and hypertension there was a higher proportion of breakfast doers, those having snacks and those not having outof-home meals.

Table 2 describe the associations between meal habits and baseline variables. Having breakfast was inversely associated with high occupational status, past smoking and moderate alcohol drinking. Older individuals were less likely to have snacks and out-ofhome meal, while women were more likely to have snacks and less likely to have out-of-home meals. Unmarried individuals with higher educational and occupational status, as well as higher physical activity and regular alcohol drinking were also more likely to have out-of-home meals. In contrast, snaking habit was associated with being married and past smoker.

Table 3 summarize the associations between meal habits and metabolic parameters. Having breakfast was associated with higher odds of having dyslipidemias $(\mathrm{OR}=2.71,95 \% \mathrm{CI}: 1.74,4.23)$; having snacks was associated with higher odds of being obese $(\mathrm{OR}=2.09,95 \% \mathrm{CI}: 1.42,3.09)$; and having out-of-home meals was associated with higher odds of having dyslipidemias $(\mathrm{OR}=1.55,95 \% \mathrm{CI}: 1.03$, 2.32) and hypertension $(\mathrm{OR}=1.61,95 \% \mathrm{CI}: 1.18$, 2.21). 
Table 1

Distribution of baseline characteristics by breakfast, snaking, and out-of-home eating features in the study sample $(n=1952)$

\begin{tabular}{|c|c|c|c|c|c|c|c|c|c|}
\hline & \multicolumn{2}{|c|}{ Breakfast } & \multirow[b]{2}{*}{$p$} & \multicolumn{2}{|c|}{ Snacks } & \multirow[b]{2}{*}{$p$} & \multicolumn{2}{|c|}{ Out-of-home eating } & \multirow[b]{2}{*}{$p$} \\
\hline & $\begin{array}{c}\text { Yes } \\
(n=1500)\end{array}$ & $\begin{array}{c}\text { No } \\
(n=452)\end{array}$ & & $\begin{array}{c}\text { Yes } \\
(n=1459)\end{array}$ & $\begin{array}{c}\text { No } \\
(n=493)\end{array}$ & & $\begin{array}{c}\text { Yes } \\
(n=956)\end{array}$ & $\begin{array}{c}\text { No } \\
(n=996)\end{array}$ & \\
\hline Age group, $n(\%)$ & & & 0.005 & & & $<0.001$ & & & $<0.001$ \\
\hline$<30$ & $265(74.0)$ & $93(26.0)$ & & $313(87.4)$ & $45(12.6)$ & & $289(80.7)$ & $19.3(6.9)$ & \\
\hline $31-39$ & $259(77.3)$ & $76(22.7)$ & & $293(87.5)$ & $42(12.5)$ & & $235(70.1)$ & $100(29.9)$ & \\
\hline $40-49$ & $272 /(73.1)$ & $100(26.9)$ & & $289(77.7)$ & $83(22.3)$ & & $196(52.7)$ & $176(47.3)$ & \\
\hline $50-59$ & $231(74.3)$ & $80(25.7)$ & & $221(71.1)$ & $90(28.9)$ & & $145(46.6)$ & $166(53.4)$ & \\
\hline $60-69$ & $252(79.7)$ & $64(20.3)$ & & $204(64.6)$ & $112(35.4)$ & & $79(25.0)$ & $237(75.0)$ & \\
\hline$>70$ & $221(85.0)$ & $39(15.0)$ & & $139(53.5)$ & $121(46.5)$ & & $12(4.6)$ & $248(95.4)$ & \\
\hline Sex & & & 0.004 & & & $<0.001$ & & & $<0.001$ \\
\hline Men & $598(73.6)$ & $215(26.4)$ & & $573(70.5)$ & $240(29.5)$ & & $485(59.7)$ & $328(40.3)$ & \\
\hline Women & $902(79.2)$ & $237(20.8)$ & & $886(77.8)$ & $253(22.2)$ & & $471(41.4)$ & $668(58.6)$ & \\
\hline Educational status & & & $<0.001$ & & & $<0.001$ & & & $<0.001$ \\
\hline Low & $578(82.2)$ & $125(17.8)$ & & $482(68.6)$ & $221(31.4)$ & & $165(23.5)$ & $538(76.5)$ & \\
\hline Medium & $524(72.1)$ & 203 (27.9) & & $562(77.3)$ & $165(22.7)$ & & $430(59.1)$ & $297(40.9)$ & \\
\hline High & $398(76.2)$ & $124(23.8)$ & & $415(79.5)$ & $107(20.5)$ & & $361(69.2)$ & $161(30.8)$ & \\
\hline Occupational status & & & 0.008 & & & $<0.001$ & & & $<0.001$ \\
\hline Unemployed & $392(83.6)$ & $77(16.4)$ & & $354(75.5)$ & $115(24.5)$ & & $182(38.8)$ & $287(61.2)$ & \\
\hline Low & $201(74.2)$ & $70(25.8)$ & & $155(57.2)$ & $116(42.8)$ & & $94(34.7)$ & $177(65.3)$ & \\
\hline Medium & $336(76.4)$ & $104(23.6)$ & & $327(74.3)$ & $113(25.7)$ & & $191(43.4)$ & $249(56.6)$ & \\
\hline High & $379(77.0)$ & $113(23.0)$ & & $373(75.8)$ & $119(24.2)$ & & $311(63.2)$ & $181(36.8)$ & \\
\hline Marital status & & & 0.294 & & & 0.598 & & & $<0.001$ \\
\hline Unmarried/single & $599(75.6)$ & $193(24.4)$ & & $587(74.1)$ & $205(25.9)$ & & $473(59.7)$ & $319(40.3)$ & \\
\hline Married/partner & $901(77.7)$ & $259(22.3)$ & & $872(75.2)$ & $288(24.8)$ & & 483 (41.6) & $677(58.4)$ & \\
\hline Smoking status & & & $<0.001$ & & & 0.003 & & & $<0.001$ \\
\hline Non-smoker & $992(82.3)$ & 214 (17.7) & & $931(77.2)$ & $275(22.8)$ & & $599(49.7)$ & $607(50.3)$ & \\
\hline Ex-smoker & $293(62.6)$ & $175(37.4)$ & & $339(72.4)$ & $129(27.6)$ & & $264(56.4)$ & $204(43.6)$ & \\
\hline Current smoker & $215(77.3)$ & $63(22.7)$ & & $189(68.0)$ & $89(32.0)$ & & $93(33.5)$ & $185(66.5)$ & \\
\hline Physical activity & & & 0.150 & & & $<0.001$ & & & $<0.001$ \\
\hline Low & $258(77.9)$ & $73(22.1)$ & & $232(70.1)$ & 99 (29.9) & & $97(29.3)$ & $234(70.7)$ & \\
\hline Moderate & $665(76.7)$ & $202(23.3)$ & & $700(80.7)$ & $167(19.3)$ & & $487(56.2)$ & $380(43.8)$ & \\
\hline High & $397(72.8)$ & $148(27.2)$ & & 403 (73.9) & $142(26.1)$ & & $340(62.4)$ & 205 (37.6) & \\
\hline Alcohol consumption & & & 0.002 & & & 0.118 & & & $<0.001$ \\
\hline None & $309(81.1)$ & $72(18.9)$ & & $271(71.1)$ & $110(28.9)$ & & $125(32.8)$ & $256(67.2)$ & \\
\hline $\begin{array}{l}\text { Moderate drinker } \\
(0.1-12 \mathrm{~g} / \mathrm{d})\end{array}$ & $902(74.2)$ & $313(25.8)$ & & $926(76.2)$ & $289(23.8)$ & & $665(54.7)$ & $550(45.3)$ & \\
\hline $\begin{array}{l}\text { Regular drinker } \\
(>12 \mathrm{~g} / \mathrm{d})\end{array}$ & $289(81.2)$ & $67(18.8)$ & & $262(73.6)$ & $94(26.4)$ & & $166(46.6)$ & $190(53.4)$ & \\
\hline \multicolumn{10}{|l|}{ BMI status } \\
\hline $\mathrm{BMI}=25-30$ & $478(76.4)$ & $148(23.6)$ & 0.270 & $456(72.8)$ & $170(27.2)$ & 0.129 & $316(50.5)$ & $310(49.5)$ & 0.072 \\
\hline $\mathrm{BMI}>30$ & $246(77.1)$ & 73 (22.9) & 0.732 & $257(80.6)$ & $62(19.4)$ & 0.011 & $91(28.5)$ & $228(71.5)$ & $<0.001$ \\
\hline Diabetes & $124(83.8)$ & $24(16.2)$ & 0.037 & $96(64.9)$ & $52(35.1)$ & 0.004 & $33(22.3)$ & $115(77.7)$ & $<0.001$ \\
\hline Dislipidemia & $317(88.1)$ & 43 (11.9) & $<0.001$ & $255(70.8)$ & $105(29.2)$ & 0.059 & $92(25.6)$ & $268(74.4)$ & $<0.001$ \\
\hline Hypertension & $780(79.3)$ & $204(20.7)$ & 0.010 & $672(68.3)$ & $312(31.7)$ & $<0.001$ & $392(39.8)$ & $592(60.2)$ & $<0.001$ \\
\hline
\end{tabular}

\section{Discussion}

The findings of this study are partially consistent with observed trends in scientific literature, showing a slow abandonment of healthy dietary habits among individuals living in southern Italy, leading to adverse metabolic-related health conditions [22-24]. Adherence to traditional healthy dietary patterns has been associated to lower risk of metabolic disorders and cardiovascular outcomes among individuals living in southern Italy [25-27], including hypertension [28], obesity [29], dyslipidemia [30], insulin resistance [31] but also overall quality of life [32]. Nevertheless, there is evidence of the so-called "nutrition transition" phenomenon, described as a shift toward Westernized dietary habits, including high-sugar, high-refined, high-fat processed foods and beverages in spite of more natural, biologic (or less processed), local goods [33, 34]; these trends have been demonstrated especially among younger generations 
Table 2

Association between baseline characteristics and breakfast, snaking, and out-of-home eating status in the study sample $(n=1952)$

\begin{tabular}{|c|c|c|c|}
\hline & \multicolumn{3}{|c|}{ OR $(95 \mathrm{CI})^{*}$} \\
\hline & Breakfast & Snacks & Out-of-home eating \\
\hline \multicolumn{4}{|l|}{ Age group, $n(\%)$} \\
\hline$<30$ & 1 & 1 & 1 \\
\hline $31-39$ & $1.00(0.63,1.58)$ & $0.79(0.47,1.32)$ & $1.10(0.66,1.86)$ \\
\hline $40-49$ & $1.11(0.69,1.78)$ & $0.36(0.22,0.59)$ & $0.65(0.39,1.08)$ \\
\hline $50-59$ & $1.00(0.61,1.64)$ & $0.27(0.16,0.45)$ & $0.26(0.15,0.43)$ \\
\hline $60-69$ & $1.14(0.70,1.85)$ & $0.22(0.13,0.36)$ & $0.13(0.07,0.21)$ \\
\hline$>70$ & $1.37(0.79,2.37)$ & $0.14(0.08,0.25)$ & $0.01(0.00,0.02)$ \\
\hline \multicolumn{4}{|l|}{ Sex } \\
\hline Men & 1 & 1 & 1 \\
\hline Women & $1.25(0.95,1.64)$ & $1.44(1.09,1.92)$ & $0.34(0.25,0.46)$ \\
\hline \multicolumn{4}{|l|}{ Educational status } \\
\hline Low & 1 & 1 & 1 \\
\hline Medium & $0.83(0.60,1.16)$ & $0.93(0.68,1.28)$ & $2.56(1.83,3.58)$ \\
\hline High & $0.89(0.59,1.33)$ & $1.00(0.67,1.48)$ & $2.69(1.82,3.98)$ \\
\hline \multicolumn{4}{|l|}{ Occupational status } \\
\hline Unemployed & 1 & 1 & 1 \\
\hline Low & $0.67(0.43,1.04)$ & $0.64(0.43,0.96)$ & $0.74(0.47,1.15)$ \\
\hline Medium & $0.55(0.37,0.81)$ & $1.13(0.77,1.66)$ & $1.32(0.88,1.97)$ \\
\hline High & $0.59(0.39,0.89)$ & $1.1180 .75,1.66)$ & $2.14(1.41,3.27)$ \\
\hline \multicolumn{4}{|l|}{ Marital status } \\
\hline Unmarried/single & 1 & 1 & 1 \\
\hline Married/partner & $1.25(0.92,1.69)$ & $1.85(1.39,2.48)$ & $0.42(0.30,0.58)$ \\
\hline \multicolumn{4}{|l|}{ Smoking status } \\
\hline Non-smoker & 1 & 1 & 1 \\
\hline Ex-smoker & $0.37(0.28,0.50)$ & $0.70(0.52,0.94)$ & $1.37(0.98,1.92)$ \\
\hline Current smoker & $0.79(0.52,1.18)$ & $1.30(0.88,1.93)$ & $1.29(0.84,1.97)$ \\
\hline \multicolumn{4}{|l|}{ Physical activity } \\
\hline Low & 1 & 1 & 1 \\
\hline Moderate & $1.29(0.90,1.85)$ & $1.39(0.98,1.99)$ & $1.81(1.23,2.66)$ \\
\hline High & $1.40(0.94,2.09)$ & $0.72(0.50,1.05)$ & $1.64(1.08,2.49)$ \\
\hline \multicolumn{4}{|l|}{ Alcohol consumption } \\
\hline None & 1 & 1 & 1 \\
\hline Moderate drinker $(0.1-12 \mathrm{~g} / \mathrm{d})$ & $0.67(0.47,0.96)$ & $0.99(0.71,1.38)$ & $2.08(1.44,3.00)$ \\
\hline Regular drinker $(>12 \mathrm{~g} / \mathrm{d})$ & $0.96(0.61,1.50)$ & $1.11(0.74,1.67)$ & $2.71(1.72,4.25)$ \\
\hline
\end{tabular}

Table 3

Association between breakfast, snaking, and out-of-home eating status and metabolic disorders in the study sample $(n=1952)$

\begin{tabular}{lccccc}
\hline & \multicolumn{5}{c}{ OR (95 CI)* } \\
\cline { 2 - 6 } & BMI $=25-30$ & BMI $>30$ & Diabetes & Dislipidemia & Hypertension \\
\hline Breakfast & $0.80(0.59,1.08)$ & $0.94(0.64,1.39)$ & $1.33(0.72,2.45)$ & $2.71(1.74,4.23)$ & $1.34(0.99,1.81)$ \\
Snacks & $0.90(0.67,1.21)$ & $2.09(1.42,3.09)$ & $1.33(0.81,2.19)$ & $1.25(0.88,1.79)$ & $1.03(0.77,1.39)$ \\
Out-of-home eating & $1.28(0.93,1.75)$ & $0.82(0.56,1.20)$ & $0.80(0.44,1.48)$ & $1.55(1.03,2.32)$ & $1.61(1.18,2.21)$ \\
\hline
\end{tabular}

*All analyses were adjusted for age, sex, educational status, marital status, smoking status, physical activity level, and alcohol consumption.

[35-37]. However, how the nutrition transition is also affecting traditional meal habits has been far less studied. Specifically, in this study we investigated the role of meal occasions outside regular meals (i.e., lunch and dinner) to test whether such habits are associated with metabolic outcomes. In this study, meal habits were associated, with a various extent, to metabolic status of adult individuals living in Sicily, southern Italy. Specifically, snaking habit was associated with higher odds of being obese, breakfast habits with having dyslipidemias, and out-of-home eating with dyslipidemias and hypertension. 
Regarding breakfast, there are several studies in scientific literature agreeing that the first meal of the day represent an important habit associated with better metabolic health [38, 39]. Therefore, public health recommendations regarding breakfast and health advice that people eat breakfast [40]. However, in line with our findings, more recent comprehensive evaluation of existing evidence suggests that eating breakfast per se, compared to skipping it, has not large impact on body weight: in contrast, size and type of breakfast seem to play a major role in affecting metabolic health [41]. For instance, highprotein breakfast (i.e., eggs) has been reported to increase weight loss compared to a high-carbohydrate breakfast (probably due to the effect in increasing levels of satiety hormones) [42, 43]. Moreover, also type of carbohydrates has been shown to influence satiety and hunger, such as high-fiber, whole grains breakfasts [44]. These factors may explain the null results found in the present study, as southern Italian breakfast is generally sweet and rarely high in protein, with no consumption of eggs or hams, as well as with poor content in fiber [45]. In our study, we found even an association between having breakfast and higher odds of having dyslipidemias. Besides the content of the specific meal, which is not likely to have a causative relation with the adverse outcome, there is evidence that other variables associated with breakfast may mediate its relation with health outcomes. For instance, breakfast may be related with regular/irregular eating outside regular meal, with higher energy intake and the resulting metabolic imbalance; having breakfast has been shown to be associated with levels of physical activity, which in fact was not observed in our study; finally, breakfast may be related with sleeping habits, which in turn has been associated with metabolic issues [46].

Snacking between meal is not univocally considered an unhealthy behavior if including healthy foods, such as nuts and whole grains [47]. In fact, snacking does not have univocal correlations with weight gain/loss and has been associated with both high and low diet quality [48]. Snacking with healthy foods may improve energy balance during the whole day and improve weight maintenance [49]. However, there is corroborated evidence that the vast majority of unhealthy outcomes associated with snacking habit depends on the poor quality of food consumed during snacking occasions, which may be energydense, nutrient-poor foods high in sodium, sugar, and/or fat (such as snack bars, salty snacks, cookies and sweets, sugar-sweetened beverages, and chips) [50]. In the present study, we found that individuals having snacks were more likely to be obese; snacking was the only meal habit directly associated with body weight, suggesting a direct relation between these variables. Several factors have been associated to the snacking habit, including hunger, location, social/food culture and environment, cognitive factors, and hedonic eating [51]. Snacking habit has been reported to have been increased over the last decades [52] and there is growing amount of scientific literature showing a relation between consumption of snacks and increase in obesity as well as other metabolic disorders [53]. Thus, quality of food consumed during snacking occasion seems to be determinant for the healthy or unhealthy role that snacking habit may have on human health.

Regarding out-of-home eating, literature on its role on weight status is contrasting, with a general increased risk of obesity features especially when associated with fast-food consumption, but also substantially null results [54]. In the present study, we did not find any association between out-of-home eating and weigh status, but there was a positive association with having dyslipidemias and hypertension, thus suggesting the possibility of poor quality food consumed during such meal occasions. Despite data on specific food consumed was not available, there is evidence from literature that meals consumed in restaurant, take away, or fast food are generally higher in energy, including abundance in free/added sugars and fats, and lower in fiber and protein [55]. Moreover, food consumed out of home has been reported to be served in large portions and be consumed in company, which is a social facilitator of food intake [56]. Considering the rising trends in out-of-home eating, the interest from the public health sector is mandatory to monitor future generations and assess quality of food services.

In terms of socio-demographic correlates, snacking and out-of-home meals decreased with older age, which is in line with scientific literature $[15,56$, 57]. Regarding sex differences, women were more likely to have snacks and less likely to have outof-home meals. These differences may reflect the socio-demographic conditions of this cohort, as a great proportion of women were unemployed, which lead to spending more time at home and thus higher availability of snacking, while men may find themselves more likely in the occasion of being out and consuming meal in working occasion or leisure time [58]. In fact, in this study married individuals were 
more likely to have snacks and less likely to consume out-of-home meals compared to unmarried/single ones. Both high education and occupational status were associated with out-of-home eating, which paired with younger age, high physical activity and higher alcohol consumption suggest that younger generations commonly adopt this meal habit. Moreover, differences in eating meals out by occupational class may suggest that such meals can be expensive as well as cultural differences in leisure activities. This information suggest that out-of-home eating does not entirely cluster with unhealthy behaviors or low socio-cultural level, but rather may be affected by low quality or excessive energy intake consequent to restaurant eating, which in turn may be related to unhealthy outcomes. This group of individuals may mostly benefit by rise in knowledge about how to eat out of home, while the food offer to the public might include healthier proposals which should combine palatability and affordable prices [59].

The present study has some limitations that need to be listed before considering conclusions. First, the cross-sectional design of the study does not allow to define causal relationships. Second, the use of FFQs, despite well-corroborated in the scientific methodology, may be affected by recall bias and underreporting. Finally, collinearity between variables or residual confounding cannot be ruled out.

In conclusions, meal habits are associated with metabolic status in adults living in the Mediterranean area. Future perspectives include rise of awareness in the general population of potential risk associated with poor quality or excessive snacking and out-of-home eating. Also, regarding breakfast, future intervention studies should aim to improve knowledge of healthy foods to be consumed in such occasion. Finally, public health efforts should be made to improve the quality of food served, and sponsoring healthy traditional food services.

\section{Acknowledgments}

The authors have no acknowledgments.

\section{Funding}

The authors report no funding.

\section{Conflict of interest}

The authors have no conflict of interest to report.

\section{References}

[1] Collaboration NCDRF. Rising rural body-mass index is the main driver of the global obesity epidemic in adults. Nature. 2019;569(7755):260-4.

[2] Collaborators GBDO, Afshin A, Forouzanfar MH, et al. Health Effects of Overweight and Obesity in 195 Countries over 25 Years. N Engl J Med. 2017;377(1):13-27.

[3] Collaborators GBDD. Health effects of dietary risks in 195 countries, 1990-2017: A systematic analysis for the Global Burden of Disease Study 2017. Lancet. 2019;393 (10184):1958-72.

[4] Angelino D, Godos J, Ghelfi F, et al. Fruit and vegetable consumption and health outcomes: An umbrella review of observational studies. Int J Food Sci Nutr. 2019;70(6): 652-67.

[5] Aune D, Keum N, Giovannucci E, et al. Nut consumption and risk of cardiovascular disease, total cancer, all-cause and cause-specific mortality: A systematic review and doseresponse meta-analysis of prospective studies. BMC Med. 2016;14(1):207.

[6] Godos J, Tieri M, Ghelfi F, et al. Dairy foods and health: An umbrella review of observational studies. Int J Food Sci Nutr. 2019:1-14

[7] Marventano S, Godos J, Tieri M, et al. Egg consumption and human health: An umbrella review of observational studies. Int J Food Sci Nutr. 2019.

[8] Grosso G, Godos J, Galvano F, Giovannucci EL. Coffee, Caffeine, and Health Outcomes: An Umbrella Review. Annu Rev Nutr. 2017;37:131-56.

[9] Keast DR, Nicklas TA, O'Neil CE. Snacking is associated with reduced risk of overweight and reduced abdominal obesity in adolescents: National Health and Nutrition Examination Survey (NHANES) 1999-2004. Am J Clin Nutr. 2010;92(2):428-35.

[10] Marangoni F, Martini D, Scaglioni S, et al. Snacking in nutrition and health. Int J Food Sci Nutr. 2019:1-15.

[11] Azemati B, Kelishadi R, Ahadi Z, et al. Association between junk food consumption and cardiometabolic risk factors in a national sample of Iranian children and adolescents population: The CASPIAN-V study. Eat Weight Disord. 2018.

[12] Payab M, Kelishadi R, Qorbani M, et al. Association of junk food consumption with high blood pressure and obesity in Iranian children and adolescents: The CASPIAN-IV Study. J Pediatr (Rio J). 2015;91(2):196-205.

[13] Keller K, Rodriguez Lopez S, Carmenate Moreno MM. Association between meal intake behaviour and abdominal obesity in Spanish adults. Appetite. 2015;92:1-6.

[14] Adams J, Goffe L, Brown T, et al. Frequency and sociodemographic correlates of eating meals out and take-away meals at home: Cross-sectional analysis of the UK national diet and nutrition survey, waves 1-4 (2008-12). Int J Behav Nutr Phys Act. 2015;12:51.

[15] Bezerra IN, Sichieri R. Eating out of home and obesity: A Brazilian nationwide survey. Public Health Nutr. 2009;12(11):2037-43. 
[16] Bes-Rastrollo M, Basterra-Gortari FJ, Sanchez-Villegas A, Marti A, Martinez JA, Martinez-Gonzalez MA. A prospective study of eating away-from-home meals and weight gain in a Mediterranean population: The SUN (Seguimiento Universidad de Navarra) cohort. Public Health Nutr. 2010;13(9): 1356-63.

[17] Grosso G, Marventano S, D’Urso M, Mistretta A, Galvano F. The Mediterranean healthy eating, ageing, and lifestyle (MEAL) study: Rationale and study design. Int J Food Sci Nutr. 2017;68(5):577-86.

[18] Marventano S, Mistretta A, Platania A, Galvano F, Grosso G. Reliability and relative validity of a food frequency questionnaire for Italian adults living in Sicily, Southern Italy. Int J Food Sci Nutr. 2016;67(7):857-64.

[19] Buscemi S, Rosafio G, Vasto S, et al. Validation of a food frequency questionnaire for use in Italian adults living in Sicily. Int J Food Sci Nutr. 2015;66(4):426-38.

[20] Mule S, Falla M, Conti A, et al. Macronutrient and Major Food Group Intake in a Cohort of Southern Italian Adults. Antioxidants (Basel). 2018;7(4).

[21] Castiglione D, Platania A, Conti A, Falla M, D'Urso M, Marranzano M. Dietary Micronutrient and Mineral Intake in the Mediterranean Healthy Eating, Ageing, and Lifestyle (MEAL) Study. Antioxidants (Basel). 2018;7(7).

[22] Grosso G, Marventano S, Buscemi S, et al. Factors associated with adherence to the Mediterranean diet among adolescents living in Sicily, Southern Italy. Nutrients. 2013;5(12): 4908-23.

[23] Grosso G, Marventano S, Giorgianni G, Raciti T, Galvano $\mathrm{F}$, Mistretta A. Mediterranean diet adherence rates in Sicily, southern Italy. Public Health Nutr. 2014;17(9): 2001-9.

[24] Bonaccio M, Bes-Rastrollo M, de Gaetano G, Iacoviello L. Challenges to the Mediterranean diet at a time of economic crisis. Nutr Metab Cardiovasc Dis. 2016;26(12): 1057-63.

[25] Marventano S, Godos J, Platania A, Galvano F, Mistretta A, Grosso G. Mediterranean diet adherence in the Mediterranean healthy eating, aging and lifestyle (MEAL) study cohort. Int J Food Sci Nutr. 2018;69(1):100-7.

[26] Grosso G, Pajak A, Mistretta A, et al. Protective role of the Mediterranean diet on several cardiovascular risk factors: Evidence from Sicily, southern Italy. Nutr Metab Cardiovasc Dis. 2014;24(4):370-7.

[27] Bonaccio M, Di Castelnuovo A, Pounis G, et al. High adherence to the Mediterranean diet is associated with cardiovascular protection in higher but not in lower socioeconomic groups: Prospective findings from the Moli-sani study. Int J Epidemiol. 2017;46(5):1478-87.

[28] La Verde M, Mule S, Zappala G, et al. Higher adherence to the Mediterranean diet is inversely associated with having hypertension: Is low salt intake a mediating factor? Int J Food Sci Nutr. 2018;69(2):235-44.

[29] Zappala G, Buscemi S, Mule S, et al. High adherence to Mediterranean diet, but not individual foods or nutrients, is associated with lower likelihood of being obese in a Mediterranean cohort. Eat Weight Disord. 2018;23(5): 605-14.

[30] Platania A, Zappala G, Mirabella MU, et al. Association between Mediterranean diet adherence and dyslipidaemia in a cohort of adults living in the Mediterranean area. Int J Food Sci Nutr. 2018;69(5):608-18.
[31] Buscemi S, Nicolucci A, Mattina A, et al. Association of dietary patterns with insulin resistance and clinically silent carotid atherosclerosis in apparently healthy people. Eur $\mathbf{J}$ Clin Nutr. 2013;67(12):1284-90.

[32] Godos J, Castellano S, Marranzano M. Adherence to a Mediterranean Dietary Pattern Is Associated with Higher Quality of Life in a Cohort of Italian Adults. Nutrients. 2019;11(5).

[33] Moreno LA, Sarria A, Popkin BM. The nutrition transition in Spain: A European Mediterranean country. Eur J Clin Nutr. 2002;56(10):992-1003.

[34] Tessier S, Gerber M. Factors determining the nutrition transition in two Mediterranean islands: Sardinia and Malta. Public Health Nutr. 2005;8(8):1286-92.

[35] Grosso G, Galvano F. Mediterranean diet adherence in children and adolescents in southern European countries. NFS Journal. 2016;3(August 2016):13-9.

[36] Iaccarino Idelson P, Scalfi L, Valerio G. Adherence to the Mediterranean Diet in children and adolescents: A systematic review. Nutr Metab Cardiovasc Dis. 2017;27(4): 283-99.

[37] Pereira-da-Silva L, Rego C, Pietrobelli A. The Diet of Preschool Children in the Mediterranean Countries of the European Union: A Systematic Review. Int J Environ Res Public Health. 2016;13(6).

[38] Betts JA, Chowdhury EA, Gonzalez JT, Richardson JD, Tsintzas K, Thompson D. Is breakfast the most important meal of the day? Proc Nutr Soc. 2016;75(4):464-74.

[39] Gibney MJ, Barr SI, Bellisle F, et al. Breakfast in Human Nutrition: The International Breakfast Research Initiative. Nutrients. 2018;10(5).

[40] Brown AW, Bohan Brown MM, Allison DB. Belief beyond the evidence: Using the proposed effect of breakfast on obesity to show 2 practices that distort scientific evidence. Am J Clin Nutr. 2013;98(5):1298-308.

[41] Dhurandhar EJ. True, true, unrelated? A review of recent evidence for a causal influence of breakfast on obesity. Curr Opin Endocrinol Diabetes Obes. 2016;23(5): 384-8.

[42] Vander Wal JS, Gupta A, Khosla P, Dhurandhar NV. Egg breakfast enhances weight loss. Int $\mathrm{J}$ Obes (Lond). 2008;32(10):1545-51.

[43] Layman DK. Protein quantity and quality at levels above the RDA improves adult weight loss. J Am Coll Nutr. 2004;23(6 Suppl):631S-6S.

[44] Maki KC, Phillips-Eakley AK, Smith KN. The Effects of Breakfast Consumption and Composition on Metabolic Wellness with a Focus on Carbohydrate Metabolism. Adv Nutr. 2016;7(3):613S-21S.

[45] Cossu M, Chiavaroli L, Dall'Asta M, Francinelli V, Scazzina F, Brighenti F. A nutritional evaluation of various typical Italian breakfast products: A comparison of macronutrient composition and glycaemic index values. Int J Food Sci Nutr. 2017:1-6.

[46] Moreno LA, Gottrand F, Huybrechts I, et al. Nutrition and lifestyle in european adolescents: The HELENA (Healthy Lifestyle in Europe by Nutrition in Adolescence) study. Adv Nutr. 2014;5(5):615S-23S.

[47] Zizza CA. Healthy snacking recommendations: One size does not fit all. Physiol Behav. 2014;134:32-7.

[48] Bellisle F. Meals and snacking, diet quality and energy balance. Physiol Behav. 2014;134:38-43. 
[49] Chapelot $D$. The role of snacking in energy balance: A biobehavioral approach. J Nutr. 2011;141(1):158-62.

[50] De Vet E, Stok FM, De Wit JB, De Ridder DT. The habitual nature of unhealthy snacking: How powerful are habits in adolescence? Appetite. 2015;95:182-7.

[51] Hess JM, Jonnalagadda SS, Slavin JL. What Is a Snack, Why Do We Snack, and How Can We Choose Better Snacks? A Review of the Definitions of Snacking, Motivations to Snack, Contributions to Dietary Intake, and Recommendations for Improvement. Adv Nutr. 2016;7(3):466-75.

[52] Zizza C, Siega-Riz AM, Popkin BM. Significant increase in young adults' snacking between 1977-1978 and 1994-1996 represents a cause for concern! Prev Med. 2001;32(4): 303-10.

[53] Berg C, Forslund HB. The Influence of Portion Size and Timing of Meals on Weight Balance and Obesity. Curr Obes Rep. 2015;4(1):11-8.

[54] Nago ES, Lachat CK, Dossa RA, Kolsteren PW. Association of out-of-home eating with anthropometric changes: A systematic review of prospective studies. Crit Rev Food Sci Nutr. 2014;54(9):1103-16.
[55] Lachat C, Nago E, Verstraeten R, Roberfroid D, Van Camp J, Kolsteren P. Eating out of home and its association with dietary intake: A systematic review of the evidence. Obes Rev. 2012;13(4):329-46.

[56] Diaz-Mendez C, Garcia-Espejo I. Eating out in Spain: Motivations, sociability and consumer contexts. Appetite. 2017;119:14-22.

[57] Kant AK, Graubard BI. Eating out in America, 1987-2000: Trends and nutritional correlates. Prev Med. 2004;38(2): 243-9.

[58] Inglis V, Ball K, Crawford D. Why do women of low socioeconomic status have poorer dietary behaviours than women of higher socioeconomic status? A qualitative exploration. Appetite. 2005;45(3):334-43.

[59] McGuffin LE, Wallace JM, McCrorie TA, Price RK, Pourshahidi LK, Livingstone MB. Family eating out-of-home: A review of nutrition and health policies. Proc Nutr Soc. 2013;72(1):126-39. 